\title{
The $\mathrm{SU}(2)$ and $\mathrm{SU}(3)$ chiral phase transitions within chiral perturbation theory
}

\author{
J. R. Peláez \\ Departamento de Física Teórica II, Universidad Complutense de Madrid, 28040 Madrid, Spain, \\ Dipartimento di Fisica, Universita' degli Studi, Firenze, Italy, \\ and INFN, Sezione di Firenze, Italy \\ (Received 10 September 2002; published 25 November 2002)
}

\begin{abstract}
The SU(2) and SU(3) chiral phase transitions in a gas made of pions, kaons, and etas are studied within the framework of chiral perturbation theory. We describe the temperature dependence of the quark condensates by using the meson meson scattering phase shifts in a second order virial expansion. In particular, the SU(3) formalism yields a somewhat lower melting temperature for the nonstrange condensates than within SU(2), and also predicts that the strange condensate melting is slower than that of the nonstrange, due to the different strange and nonstrange quark masses.
\end{abstract}

DOI: 10.1103/PhysRevD.66.096007

PACS number(s): 11.30.Rd, 11.30.Qc, 12.38.Aw, 12.39.Fe

There is a growing interest in studying the phase diagram of QCD, namely, the transition from a hadron gas to a quark gluon plasma, the chiral phase transitions, their dependence on the number of flavors, on quark masses, possible color superconducting states, etc.

The virial expansion is a simple and successful approach to describe many thermodynamic features of dilute gases made of interacting pions [1] and other hadrons [2]. Remarkably, for most thermodynamic properties it is enough to know the low energy scattering phase shifts of the particles, which could be taken from experiment, avoiding any model dependence. However, if one is interested in chiral symmetry restoration, whose order parameters are the quark condensates, defined as derivatives of the pressure with respect to the quark masses, one needs a theoretical description of the mass dependence of the scattering amplitudes.

With that aim we turn to chiral perturbation theory (ChPT) [3-5], which provides a systematic description of low energy hadronic interactions. After identifying the pions, kaons and the eta as the Goldstone bosons of the QCD spontaneous chiral symmetry breaking (pseudo Goldstone bosons, due to the small light-quark masses), the ChPT Lagrangian is built as the most general derivative and mass expansion, over $4 \pi F \simeq 1.2 \mathrm{GeV}$ (the symmetry breaking scale), respecting the symmetry constraints. At one loop any calculation can be renormalized in terms of just a set of parameters, $L_{k}(\mu), H_{k}(\mu)$ ( $\mu$ being the renormalization scale), which can be determined from a few experiments and used for further predictions at low temperatures.

In particular, within $\mathrm{SU}(2) \mathrm{ChPT}$ the leading coefficients of the low temperature expansion for the pressure and the quark condensate $\langle\bar{q} q\rangle=\langle\bar{u} u+\bar{d} d\rangle$ in a hadron gas whose only interacting hadrons were the pions and the other hadrons were free, have been calculated [6] obtaining also an estimate, $T \simeq 190 \mathrm{MeV}$, of the phase transition critical temperature. It was also shown that the perturbative calculation of the pressure was analogous to the second order virial expansion when the interacting part of the second virial coefficient is obtained from the one loop $\pi \pi$ ChPT scattering lengths. Other works have studied the applicability of the virial expansion with a chemical potential [7], or the critical temperature in generalized scenarios [8].
The SU(2) approach is limited by the absence of other interacting particles, such as kaons, whose densities at $T$ $\simeq 150 \mathrm{MeV}$ are significant [6]. Let us recall that the chiral phase transition can be different for the $\mathrm{SU}(2)$ and $\mathrm{SU}(3)$ cases, and that several QCD inequalities and lattice results suggest a chiral condensate temperature suppression with an increasing number of light flavors [9], that is, entropy and disorder. This effect would lower the chiral critical temperature down by roughly $20 \mathrm{MeV}$ [10] (in the chiral limit). Note that none of these results have been obtained from the hadronic phase.

In addition, within $\mathrm{SU}(2)$ it is not possible to study the $\langle\bar{s} s\rangle$ condensate. Furthermore, in the chiral phase diagram, quark masses play the same role as magnetic fields in ferromagnets: Intuitively, we need a higher temperature to disorder a ferromagnet when there is a magnetic field aligned along the direction of the magnetization. Analogously, it has been found that the SU(2) chiral condensate melts at a lower temperature in the massless limit [6]. Hence, since the strange quark mass $m_{s}>m_{u}, m_{d}$, we expect a "ferromagnetic" effect and a slower temperature evolution for $\langle\bar{s} s\rangle$ than for $\langle\bar{q} q\rangle$. Using a QCD-motivated effective Lagrangian in the large $N_{c}$ limit, this effect has been studied in [11]

In this work we will use SU(3) ChPT together with the virial expansion to estimate the effect on $\langle\bar{q} q\rangle$ of pions interacting with kaons and etas, and study the effect of the different quark masses. In particular, we will obtain the temperature dependence of $\langle\bar{s} s\rangle$ in the hadronic phase.

Thus, the second order relativistic virial expansion of the pressure for an inhomogeneous gas made of three species, $i$ $=\pi, K, \eta$ is $[1,12]$

$$
\beta P=\sum_{i} B_{i}(T) \xi_{i}+\sum_{i}\left(B_{i i} \xi_{i}^{2}+\frac{1}{2} \sum_{j \neq i} B_{i j} \xi_{i} \xi_{j}\right) \ldots
$$

where $\beta=1 / T$ and $\xi_{i}=\exp \left(-\beta m_{i}\right)$. Expanding up to the second order in $\xi_{i}$ means that we consider only binary interactions. For a free boson gas, $B_{i j}^{(0)}=0$ for $i \neq j$, whereas 


$$
\begin{aligned}
& B_{i}^{(0)}=\frac{g_{i}}{2 \pi^{2}} \int_{0}^{\infty} d p p^{2} e^{-\beta\left(\sqrt{p^{2}+m_{i}^{2}}-m_{i}\right)}, \\
& B_{i i}^{(0)}=\frac{g_{i}}{4 \pi^{2}} \int_{0}^{\infty} d p p^{2} e^{-2 \beta\left(\sqrt{p^{2}+m_{i}^{2}}-m_{i}\right)},
\end{aligned}
$$

and the degeneracy is $g_{i}=3,4,1$ for $\pi, K, \eta$, respectively. The interactions appear through $[6,12]$

$$
B_{i j}^{(i n t)}=\frac{\xi_{i}^{-1} \xi_{j}^{-1}}{2 \pi^{3}} \int_{m_{i}+m_{j}}^{\infty} d E E^{2} K_{1}(E / T) \Delta^{i j}(E),
$$

where $K_{1}$ is the first modified Bessel function and

$$
\Delta^{i j}=\sum_{I, J, S}(2 I+1)(2 J+1) \delta_{I, J, S}^{i j}(E)
$$

$\delta_{I, J, S}^{i j}$ being the $i j \rightarrow i j$ phase shifts (chosen so that $\delta=0$ at threshold) of the elastic scattering of a state $i j$ with quantum numbers $I, J, S$ ( $J$ being the total angular momentum and $S$ the strangeness).

The virial expansion seems to break [7] at $T \simeq 200$ $-250 \mathrm{MeV}$. Fortunately, the physics we are interested in occurs already at $T<250 \mathrm{MeV}$, and $\xi_{\pi}>\xi_{K} \simeq \xi_{\eta}$, so that it is enough to consider $i j=\pi \pi, \pi K$ and $\pi \eta$ in the second virial coefficients. Thus we are considering up to $\xi_{\pi}$ suppressed corrections to the $\pi, K, \eta$ dilute free gas.

Let us recall that the pressure is nothing but the $T$ dependent part of the free energy density $z$, i.e., $P=\epsilon_{0}-z, \epsilon_{0}$ being the vacuum energy density. Hence [6],

$$
\left\langle\bar{q}_{\alpha} q_{\alpha}\right\rangle=\frac{\partial z}{\partial m_{q_{\alpha}}}=\left\langle 0\left|\bar{q}_{\alpha} q_{\alpha}\right| 0\right\rangle-\frac{\partial P}{\partial m_{q_{\alpha}}},
$$

where $q_{\alpha}=u, d, s$. Let us emphasize that, in contrast with most thermodynamic quantities, for the chiral condensate it is not enough to know the $\delta(E)$, but we also need their dependence with the quark masses as well as a value for the vacuum expectation value. All that will be obtained from ChPT. For that purpose, we first rewrite the condensate, Eq. (6), in terms of meson masses:

$$
\left\langle\bar{q}_{\alpha} q_{\alpha}\right\rangle=\left\langle 0\left|\bar{q}_{\alpha} q_{\alpha}\right| 0\right\rangle\left(1+\sum_{i} \frac{c_{i}^{\bar{q}_{\alpha} q_{\alpha}}}{2 m_{i} F^{2}} \frac{\partial P}{\partial m_{i}}\right)
$$

where, as before, $i=\pi, K, \eta$. Note that we have defined

$$
c_{i}^{\bar{q}_{\alpha} q_{\alpha}}=-F^{2} \frac{\partial m_{i}^{2}}{\partial m_{q_{\alpha}}}\left\langle 0\left|\bar{q}_{\alpha} q_{\alpha}\right| 0\right\rangle^{-1} .
$$

In the isospin limit, when $m_{u}=m_{d}$, the $u$ and $d$ condensates are equal, and we define $\langle 0|\bar{q} q| 0\rangle \equiv\langle 0|\bar{u} u+\bar{d} d| 0\rangle$. It is tedious but straightforward to obtain expressions for the $c$ parameters above. They can be found in the Appendix. Both the $\langle 0|\bar{q} q| 0\rangle$ and $\langle 0|\bar{s} s| 0\rangle$ one loop calculations within $\mathrm{SU}(3)$ ChPT were given in [5], together with the one loop dependence of the meson masses on the quark masses needed to
TABLE I. Different sets of chiral parameters $\times 10^{3}$. In the second column $L_{1}^{r}, L_{2}^{r}, L_{3}$ are taken from [22] and the rest from [4] $\left(L_{4}^{r}\right.$ and $L_{6}^{r}$ are estimated from the Zweig rule). The third column comes from an $O\left(p^{4}\right)$ analysis of $K_{l 4}$ decays [21] $\left(L_{4}^{r}\right.$ and $L_{6}^{4}$ are set to zero). The last column is the IAM fit [17] to the whole mesonmeson scattering up to $1.2 \mathrm{GeV}$.

\begin{tabular}{lrcc}
\hline \hline & Refs. [4,22] & Ref. [21] & IAM [17] \\
\hline$L_{1}^{r}\left(M_{\rho}\right)$ & $0.4 \pm 0.3$ & 0.46 & $0.561 \pm 0.008( \pm 0.10)$ \\
$L_{2}^{r}\left(M_{\rho}\right)$ & $1.35 \pm 0.3$ & 1.49 & $1.21 \pm 0.001( \pm 0.10)$ \\
$L_{3}$ & $-3.5 \pm 1.1$ & -3.18 & $-2.79 \pm 0.02( \pm 0.12)$ \\
$L_{4}^{r}\left(M_{\rho}\right)$ & $-0.3 \pm 0.5$ & 0 & $-0.36 \pm 0.02( \pm 0.17)$ \\
$L_{5}^{r}\left(M_{\rho}\right)$ & $1.4 \pm 0.5$ & 1.46 & $1.4 \pm 0.02( \pm 0.5)$ \\
$L_{6}^{r}\left(M_{\rho}\right)$ & $-0.2 \pm 0.3$ & 0 & $0.07 \pm 0.03( \pm 0.08)$ \\
$L_{7}$ & $-0.4 \pm 0.2$ & -0.49 & $-0.44 \pm 0.003( \pm 0.15)$ \\
$L_{8}^{r}\left(M_{\rho}\right)$ & $0.9 \pm 0.3$ & 1.00 & $0.78 \pm 0.02( \pm 0.18)$ \\
\hline \hline
\end{tabular}

obtain $\partial m_{i}^{2} / \partial m_{q_{\alpha}}$. The only relevant comment is that the $c$ coefficients depend on the chiral parameters $L_{k}$, for $k$ $=4 \ldots 8$, and $\mathrm{H}_{2}$.

In Table I, we show several $L_{k}$ determinations from meson data. It makes little difference to use other determinations. For $H_{2}$ we will use $H_{2}^{r}\left(M_{\rho}\right)=(-3.4 \pm 1.1) 10^{-3}$, obtained as explained in [13] but using a more recent estimation of $\langle 0|\bar{s} s| 0\rangle /\langle 0|\bar{q} q| 0\rangle=0.75 \pm 0.12$ [14]. For instance, the $c$ parameters obtained when using the $L_{k}$ in the first column of Table I are

$$
\begin{aligned}
c_{\pi}^{\bar{q} q} & =0.9_{-0.4}^{+0.2}, c_{K}^{\bar{q} q}=0.5_{-0.7}^{+0.4}, c_{\eta}^{\bar{q} q}=0.4_{-0.7}^{+0.5}, \\
c_{\pi}^{\bar{s} s} & =-0.005_{-0.037}^{+0.029}, c_{K}^{\bar{s} s}=1.3_{-0.8}^{+0.4}, c_{\eta}^{\bar{s} s}=1.5_{-1.6}^{+0.9} .
\end{aligned}
$$

Note that our $c_{\pi}^{\bar{q} q}$ is in a good agreement with the $\mathrm{SU}(2)$ estimates: 0.85 and $0.90 \pm 0.05[6]$. Had we used the estimate $H_{2}^{r}\left(M_{\rho}\right) \simeq 2 L_{8}^{r}\left(M_{\rho}\right) \simeq 1.8 \times 10^{-3}$ from scalar resonance saturation [15], all our following results would remain unchanged for $\langle\bar{q} q\rangle$, but the strange condensate would not even seem to melt within this approximation. Finally, we need the meson-meson amplitudes to one-loop in SU(3) ChPT, which have been given in $[16,17]$. These expressions have to be projected in partial waves of definite isospin $I$ and angular momentum $J$, whose complex phases are the $\delta_{I, J}(E)$ in Eq. (5). ChPT is known to provide a good low energy description $(E<500 \mathrm{MeV})$ of the meson-meson amplitudes [16]. Within $\mathrm{SU}(2) \mathrm{ChPT}$ it has been shown [6] that using just the amplitudes at threshold (scattering lengths) in the virial expansion is equivalent to expanding the partition function to third order in $T / F$ or $T / m_{\pi}$. This approach yields a fairly good representation of the pion gas thermodynamics at low temperatures, $T<150 \mathrm{MeV}$ [6].

The virial coefficients and $\partial P / \partial m_{i}$ have been calculated numerically. For the figures, we have represented the chiral condensate over its vacuum expectation value, $\left\langle\bar{q}_{\alpha} q_{\alpha}\right\rangle /\left\langle 0\left|\bar{q}_{\alpha} q_{\alpha}\right| 0\right\rangle$, so that all of them are normalized to 1 at $T=0$. In reality, since there is always a small explicit chiral symmetry breaking due to the quark masses, the con- 


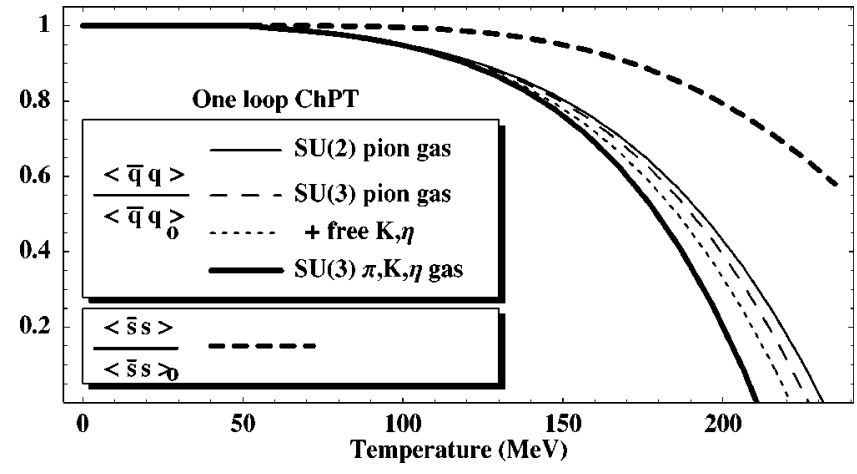

FIG. 1. Condensate evolution with temperature using one-loop ChPT amplitudes. Although they should not really vanish they are extrapolated down to zero only for reference.

densate does not vanish completely, except in the $T \rightarrow \infty$ limit (following with our analogy, our ferromagnet above the $\mathrm{Cu}$ rie point becomes paramagnetic in the presence of a magnetic field). Of course, with the second order virial approach we cannot generate such an analytic behavior, and our curves become negative above some $T$. Since the nonstrange quark masses are very tiny compared with the $\langle\bar{q} q\rangle$ (about 3\%), it is a good approximation to let the condensate vanish and talk about a melting temperature. This is not the case for the strange condensate, which has a larger explicit breaking due to the much larger mass of this quark.

In Fig. 1 we show the results of using the one-loop ChPT expansion for the phase shifts. The thin continuous line represents the interacting pion gas in $\mathrm{SU}(2)$, but updated to the $\mathrm{SU}(2)$ parameters obtained from those in the first column of Table I (see [5] for the conversion), whereas the thin-dashed line is the corresponding one-loop $\mathrm{SU}(3)$ result also for a pion gas. We have checked that the tiny difference between them comes only from the $O\left(p^{4}\right)$ phase shift contribution [it is the same for $\mathrm{SU}(2)$ and $\mathrm{SU}(3)$ only up to $s / M_{K}^{2}$ or $s / M_{\eta}^{2}$ terms from expanding kaon or eta loops [5]]. This amounts to a $4 \mathrm{MeV}$ decrease of the pion gas extrapolated melting temperature: $\quad T_{m}^{\langle\bar{q} q\rangle}=231 \mathrm{MeV}$ for $\mathrm{SU}(2)$ and $T_{m}^{\langle\bar{q} q\rangle}$ $=227 \mathrm{MeV}$ for $\mathrm{SU}(3)$. Next, the thin-dotted line is obtained by adding free kaons and etas to the pion gas, which lowers the extrapolated melting temperature down to $221 \mathrm{MeV}$.

Our new $\mathrm{SU}(3)$ result for a $\pi, K, \eta$ gas, is the thick continuous line, where we see that the additional decrease due to $\pi K, \pi \eta$ interactions amounts to 10 or $11 \mathrm{MeV}$. It may seem surprising that the $\pi K, \pi \eta$ interaction effects may be comparable to or larger than that of free kaons and etas, since they are thermally suppressed. Note, however, that exp $\left(-m_{\pi} / T\right)$ just amounts to a factor of 6,4,2.5 suppression at $T=80,100,150 \mathrm{MeV}$, respectively. In contrast, the $\pi K$ and $\pi \eta$ interactions depend strongly on $m_{\pi}$, much more sensitive to $\hat{m}$ than $m_{K}$ or $m_{\eta}$, which are the only dependence of the free $K$ and $\eta$ terms on $\hat{m}$. In particular $\partial m_{\pi}^{2} / \partial \hat{m}$ $\simeq 2 \partial m_{K}^{2} / \partial \hat{m}$ and $\partial m_{\pi} / \partial \hat{m} \simeq 6 \partial m_{K} / \partial \hat{m}$, and this "temperature independent enhancement" competes with the thermal suppression, making the $\pi K$ and $\pi \eta$ interaction effects comparable to the free one around $T=80-100 \mathrm{MeV}$ and larger if $T>140 \mathrm{MeV}$. All together, the $T_{m}^{\langle\bar{q} q\rangle}$ decrease in SU(3) is roughly $20 \mathrm{MeV}$, in agreement with the chiral limit lattice results $T_{c}=173 \mathrm{MeV}$ for $\mathrm{SU}(2)$ and $T_{c}=154 \mathrm{MeV}$, for $\mathrm{SU}(3)[10]$.

Furthermore, it can be noticed that $\langle\bar{s} s\rangle$ melts much slower than $\langle\bar{q} q\rangle$, since $m_{s} \gg \hat{m}$. Indeed, there is still $70 \%$ left of the $\langle\bar{s} s\rangle$ condensate at the $\langle\bar{q} q\rangle$ melting point. Let us recall that both effects are already sizable at low temperatures $T \simeq 100 \mathrm{MeV}$, even lower when looking at the slower $\langle\bar{s} s\rangle$ evolution, which already separates from the $\langle\bar{q} q\rangle$ evolution at temperatures as low as $70 \mathrm{MeV}$. Since we can still trust pure ChPT up to, roughly, $T \simeq 150 \mathrm{MeV}$ [6], we conclude that both effects are model independent predictions.

However, just for illustration and to ease the comparison between curves and with previous works, we have extrapolated the condensates to higher temperatures. We have also made a Monte Carlo Gaussian sampling of the chiral parameters within their error bands. We find that the melting temperatures would be

$$
\begin{aligned}
T_{m}^{\langle\bar{q} q\rangle S U(3)} & =211_{-7}^{+19} \mathrm{MeV}, \\
T_{m}^{\langle\bar{q} q\rangle S U(2)} & =231_{-10}^{+30} \mathrm{MeV}, \\
T_{m}^{\langle\bar{q} q\rangle S U(2)}-T_{m}^{\langle\bar{q} q\rangle S U(3)} & =21_{-7}^{+14} \mathrm{MeV} .
\end{aligned}
$$

Note that the two melting temperatures are strongly correlated.

Concerning the $\langle\bar{s} s\rangle$ extrapolation, its "melting" temperature $T_{m}^{\langle\bar{s} s\rangle}=291_{-35}^{+37} \mathrm{MeV}$, is just for illustration, since, apart from lying beyond the reliability region of the approach, as stated above, actually it should not melt completely, except in the $T \rightarrow \infty$ limit. In particular, we would find $T_{m}^{\langle\bar{s} s\rangle}-T_{m}^{\langle\bar{q} q\rangle}$ $=80_{-40}^{+25} \mathrm{MeV}$. Nevertheless, we remark once more that the slower $\langle\bar{s} s\rangle$ thermal evolution is clearly seen at temperatures where the whole approach can be trusted. As a matter of fact, from the figure, we see that the strange condensate does not start to melt sizably up to $T \simeq 150 \mathrm{MeV}$.

Furthermore, we have also estimated the effect of other, more massive, hadrons, which in the $\mathrm{SU}(2)$ case $[6,7]$ also included the kaons and etas, and reduced $T_{m}^{\langle\bar{q} q\rangle}$ by approximately 10-20 MeV. Since they are heavier than $m_{\eta}$, their density is very low, and their main contribution to the pressure comes from the first virial coefficient, i.e. the free gas. The only uncertainty is on $\partial M_{h} / \partial m_{q_{\alpha}}$, conservatively estimated to lie within the number of valence quarks $N_{q_{\alpha}}$ and $2 N_{q_{\alpha}}$. Thus, we have found that their contribution decreases $T_{m}^{\langle\bar{q} q\rangle}$ by 7-12 MeV, respectively. The heavy hadron contribution is the same for the $\mathrm{SU}(2)$ and $\mathrm{SU}(3)$ cases, so that the difference between their respective melting temperatures remains the same. Finally if we were to extrapolate the strange condensate evolution we would also find a decrease of $T_{m}^{\langle s s\rangle}$ by $15-23 \mathrm{MeV}$ due to these heavier hadrons.

As an attempt to estimate the uncertainty due to the truncation of the virial expansion, we have added to our calculation the third order coefficient for free pions, which can be calculated exactly. If we do so, the melting temperature de- 


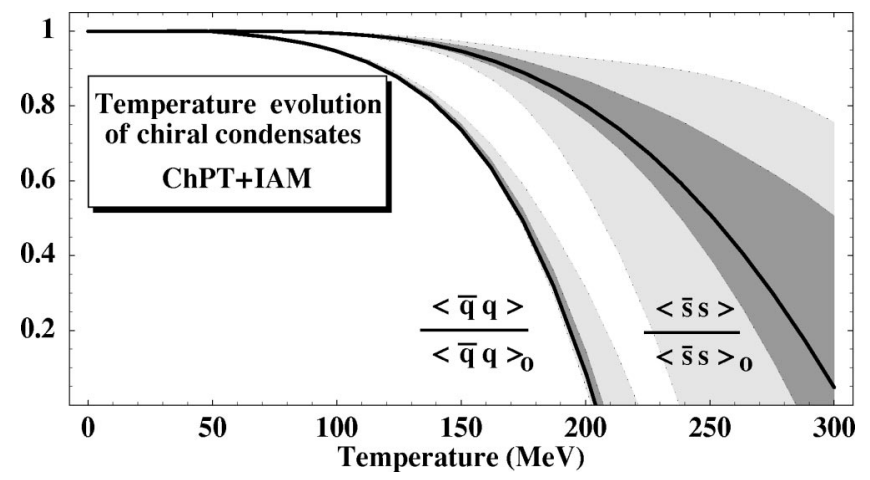

FIG. 2. Temperature evolution of chiral condensates using the unitarized ChPT amplitudes. The shaded areas cover the uncertainties in different sets of chiral parameters. Although they should not really vanish they are extrapolated down to zero only to ease their comparison and for reference.

creases by $1 \mathrm{MeV}$. It seems that when higher order corrections start becoming important, the curve is already so steep that the melting point changes very slightly. We cannot calculate the size of the three pion interaction term, but we expect it to be of the same order, and consistently with what we did for the heavy hadron uncertainty, we also allow for twice as much uncertainty, i.e., another $2 \mathrm{MeV}$. The important point, however, is that the error is dominated by the uncertainty in the chiral parameters, which is an order of magnitude larger. Altogether, we find

$$
T_{m}^{\langle\bar{q} q\rangle} \simeq 201_{-11}^{+23} \mathrm{MeV} .
$$

Finally, and in order to estimate the effects of higher energies we can extend ChPT up to $E \simeq 1.2 \mathrm{GeV}$ by means of unitarization models [17-19]. These techniques resum the ChPT series respecting unitarity but also the low energy expansion, including the mass dependency. In particular, it has been shown that the coupled channel inverse amplitude method (IAM) provides a remarkable and accurate description of the complete meson-meson interactions below 1.2 $\mathrm{GeV}$, generating dynamically six resonances from the oneloop ChPT expansion and a set of fitted $L_{k}$ compatible with other previous determinations. This approach can be extended systematically to higher orders [for the $\mathrm{SU}(2)$ case, see [20]].

Hence, in Fig. 2 we show the results of using the one-loop coupled channel IAM fitted phase shifts. Its corresponding $L_{i}$ parameters are given in the last column of Table I, with two errors: the first, very small, is purely statistical, and the second covers the uncertainty in the parameters depending on what systematic error is assumed for the experimental data. Let us remark that, although the $L_{k}$ are highly correlated, the second, larger error, ignores completely these correlations, and should be considered as a very conservative range. The continuous line corresponds to the central values, and the dark shaded areas cover the one standard deviation uncertainty due to the small errors in the parameters. These areas have been obtained from a Monte Carlo Gaussian sampling. The conservative ranges are covered by the light gray areas. We now find

$$
\begin{aligned}
T_{m}^{\langle\bar{q} q\rangle S U(3)} & =204_{-1}^{+3}\left({ }_{5}^{13}\right) \mathrm{MeV}, \\
T_{m}^{\langle\bar{q} q\rangle S U(2)} & =235_{-1}^{+3}\left({ }_{1}^{14}\right) \mathrm{MeV}, \\
T_{m}^{\langle\bar{q} q\rangle S U(2)}-T_{m}^{\langle\bar{q} q\rangle S U(3)} & =31.50_{-0.03}^{+1.20}\left({ }_{8}^{9}\right) \mathrm{MeV}
\end{aligned}
$$

where the errors in parentheses are the conservative errors which should be interpreted as uncertainty ranges better than as standard deviations. Note the excellent agreement with standard ChPT. The magnitude of the different contributions is roughly the same, although the $\pi K$ and $\pi \eta$ interactions this time lower $T_{m}^{\langle\bar{q} q\rangle}$ by $17 \mathrm{MeV}$, and the free kaons and etas by roughly $10 \mathrm{MeV}$. Let us remark that the strange condensate again melts much slower than the nonstrange condensate, and that this effect is clearly sizable at low temperatures, where both ChPT and the virial expansion can be trusted. With all the caveats presented above, and just for illustration we also extrapolate $\langle\bar{s} s\rangle$ to zero and we find $T_{m}^{\langle\bar{s} s\rangle}=304_{-25}^{+39}\left(\begin{array}{c}120 \\ 65\end{array}\right) \mathrm{MeV}$, in particular, the strange condensate melting is retarded by $T_{m}^{\langle\bar{s} s\rangle}-T_{m}^{\langle\bar{q} q\rangle}=100_{-29}^{+36}\left({ }_{80}^{120}\right) \mathrm{MeV}$. Again other massive states apart from kaons and etas would lower $T_{m}^{\langle\bar{q} q\rangle}$ by $6-10 \mathrm{MeV}$, and $T_{m}^{\langle\bar{s} s\rangle}$ by $22-32 \mathrm{MeV}$. Also very similar is the effect of including the third order virial coefficient for free pions. As it happened in the standard ChPT case, it decreases the melting temperature by $1 \mathrm{MeV}$, and we add another $2 \mathrm{MeV}$ of uncertainty for the unknown interacting part, that is

$$
T_{m}^{\langle\bar{q} q\rangle} \simeq 195_{-5}^{+8}\left({ }_{7}^{17}\right) \mathrm{MeV} .
$$

Again, the errors correspond to the uncertainty in the chiral parameters plus that on the heavier states, plus that from the truncation of the virial expansion and those in parentheses should be interpreted as conservative ranges.

These results show that higher energy effects do not affect very much our conclusions and that the results of the integrals in Eq. (4), which extend to infinity, seem robust.

We have studied the $\mathrm{SU}(2)$ and $\mathrm{SU}(3)$ temperature evolution of the chiral condensates in the hadronic phase. The thermodynamics of the meson gas has been obtained from the virial expansion and chiral perturbation theory. Our results clearly show a significant decrease, about $20 \mathrm{MeV}$, of the nonstrange condensate melting temperature, from the $\mathrm{SU}(2)$ to the $\mathrm{SU}(3)$ case, similar to lattice results. Of these, about $6 \mathrm{MeV}$ had already been explained with free kaons and etas, but the rest are mainly due to $\pi K$ and $\pi \eta$ interactions. In addition, our results show a slower temperature evolution of the strange condensate, about $90 \%$ of its zero value still remaining at $150 \mathrm{MeV}$, due to the different quark masses. Both effects are clearly visible already at low temperatures. However, we have checked that their size is completely similar when using unitarized models.

These techniques should be easily extended to heavy baryon chiral perturbation theory, in order to study the condensates with nonzero baryon density. In general the whole approach could be used with any effective Lagrangian formalism, and in particular to study other QCD phase transitions like those of the color superconducting phases. 


\section{APPENDIX}

We provide here the expressions for the $\mathrm{SU}(3) c_{i}$ coefficients:

$$
\begin{aligned}
& c_{\pi}^{\bar{q} q}=1+4 \mu_{\pi}+2 \mu_{K}+m_{\pi}^{2}\left(\nu_{\pi}-\frac{\nu_{K}}{9}\right)-\frac{16 m_{K}^{2}}{F_{0}^{2}} L_{4}+\frac{4 m_{\pi}^{2}}{F_{0}^{2}}\left(8 L_{6}+6 L_{8}-6 L_{4}-4 L_{5}-H_{2}\right) \\
& c_{K}^{\bar{q} q}=\frac{1}{2}+\frac{3 \mu_{\pi}}{2}+\mu_{K}+\frac{\mu_{\eta}}{2}-\frac{4 m_{\pi}^{2}}{F_{0}^{2}}\left(L_{4}+L_{8}+\frac{H_{2}}{2}\right)+\frac{2 m_{K}^{2} \nu_{\eta}}{9}+\frac{8 m_{K}^{2}}{F_{0}^{2}}\left(4 L_{6}+2 L_{8}-3 L_{4}-L_{5}\right) \\
& c_{\eta}^{\bar{q} q}=\frac{1}{3}\left\{1+6 \mu_{K}-3 m_{\pi}^{2} \nu_{\pi}+\frac{\nu_{\eta}}{3}\left(m_{\pi}^{2}-4 m_{\eta}^{2}\right)+\nu_{K}\left(3 m_{\eta}^{2}+m_{\pi}^{2}\right)+\frac{16 m_{\eta}^{2}}{F_{0}^{2}}\left(6 L_{6}+2 L_{8}-L_{5}-3 L_{4}\right)\right. \\
& \left.-\frac{16 L_{4}}{F_{0}^{2}}\left(m_{K}^{2}+m_{\pi}^{2} / 2\right)-\frac{128}{3 F_{0}^{2}}\left(m_{K}^{2}-m_{\pi}^{2}\right)\left(3 L_{7}+L_{8}\right)-\frac{4 m_{\pi}^{2}}{F_{0}^{2}}\left(2 L_{8}+H_{2}\right)\right\} \\
& c_{\pi}^{\overline{s s}}=\frac{16 m_{\pi}^{2}}{F_{0}^{2}}\left(2 L_{6}-L_{4}\right)-\frac{4}{9} \nu_{\eta} m_{\pi}^{2} \\
& c_{K}^{\overline{s s}}=\left\{1+2 \mu_{\eta}+4 \mu_{K}+\frac{4 m_{\pi}^{2}}{F_{0}^{2}}\left[2\left(L_{8}-L_{4}\right)+H_{2}\right]+\frac{8}{9} \nu_{\eta} m_{K}^{2}+\frac{16 m_{K}^{2}}{F_{0}^{2}}\left(2 L_{6}+L_{8}-2 L_{4}-L_{5}-H_{2} / 2\right)\right\} \\
& c_{\eta}^{\overline{s s}}=\frac{4}{3}\left\{1+6 \mu_{K}+m_{\eta}^{2}\left(3 \nu_{K} / 2-4 \nu_{\eta} / 3\right)+m_{\pi}^{2}\left(\nu_{K} / 2+\nu_{\eta} / 3\right)+\frac{16 m_{K}^{2}}{F_{0}^{2}}\left(4 L_{7}-L_{4}-\frac{H_{2}}{2}+\frac{L_{8}}{3}\right)\right. \\
& \left.+\frac{8 m_{\pi}^{2}}{F_{0}^{2}}\left(-L_{4}-\frac{5}{3} L_{8}+\frac{H_{2}}{2}-8 L_{7}\right)+\frac{8 m_{\eta}^{2}}{F_{0}^{2}}\left(4 L_{8}-2 L_{5}-\frac{3 L_{4}}{2}+3 L_{6}\right)\right\}
\end{aligned}
$$

where following the standard notation in Ref. [5] $\mu_{i}=m_{i}^{2} \log \left(m_{i}^{2} / \mu^{2}\right) /\left(32 \pi^{2} F_{0}^{2}\right), \nu=\left[\log \left(m_{i}^{2} / \mu^{2}\right)+1\right] /\left(32 \pi^{2} F_{0}^{2}\right)$ and $F_{0}$ is the pion decay constant in the $\mathrm{SU}(3)$ chiral limit. In this work we have set the renormalization scale to $\mu=M_{\rho}$.

The author thanks A. Dobado, A. Gómez Nicola and E. Oset for useful comments, and support from the Spanish CICYT projects FPA2000 0956 and BFM2000 1326, as well as the Marie Curie fellowship through Grant No. MCFI-2001-01155.

[1] G.M. Welke, R. Venugopalan, and M. Prakash, Phys. Lett. B 245, 137 (1990); V.L. Eletsky, J.I. Kapusta, and R. Venugopalan, Phys. Rev. D 48, 4398 (1993).

[2] R. Venugopalan and M. Prakash, Nucl. Phys. A546, 718 (1992).

[3] S. Weinberg, Physica A 96, 327 (1979).

[4] J. Gasser and H. Leutwyler, Ann. Phys. (N.Y.) 158, 142 (1984).

[5] J. Gasser and H. Leutwyler, Nucl. Phys. B250, 465 (1985); B250, 517 (1985); B250, 539 (1985).

[6] P. Gerber and H. Leutwyler, Nucl. Phys. B321, 387 (1989).

[7] A. Dobado and J.R. Peláez, Phys. Rev. D 59, 034004 (1999).

[8] J.R. Peláez, Phys. Rev. D 59, 014002 (1999).

[9] R.D. Mawhinney, Nucl. Phys. B (Proc. Suppl.) A60, 306 (1998); C. Sui, ibid. 73, 228 (1999); B. Moussallam, Eur. Phys. J. C 14, 111 (2000).

[10] F. Karsch, A. Peikert, and E. Laermann, Nucl. Phys. B605, 579 (2001).

[11] T. Hatsuda and T. Kunihiro, Phys. Lett. B 198, 126 (1987).

[12] R. Dashen, S. Ma, and H.J. Bernstein, Phys. Rev. 187, 187 (1969).
[13] M. Jamin, Phys. Lett. B 538, 71 (2002).

[14] S. Narison, hep-ph/0202200.

[15] G. Ecker et al., Nucl. Phys. B321, 311 (1989).

[16] V. Bernard, N. Kaiser, and U.-G. Meißner, Phys. Rev. D 43, 2757 (1991); 44, 3698 (1991); Nucl. Phys. B357, 129 (1991).

[17] A. Gómez Nicola and J.R. Peláez, Phys. Rev. D 65, 054009 (2002).

[18] T.N. Truong, Phys. Rev. Lett. 61, 2526 (1988); 67, 2260 (1991); A. Dobado, M.J. Herrero, and T.N. Truong, Phys. Lett. B 235, 134 (1990); A. Dobado and J.R. Peláez, Phys. Rev. D 47, 4883 (1993); 56, 3057 (1997).

[19] J.A. Oller, E. Oset, and J.R. Peláez, Phys. Rev. Lett. 80, 3452 (1998); Phys. Rev. D 59, 074001 (1999); 60, 099906(E) (1999); F. Guerrero and J.A. Oller, Nucl. Phys. B537, 459 (1999); B602, 641(E) (2001).

[20] J. Nieves, M. Pavón Valderrama, and E. Ruiz Arriola, Phys. Rev. D 65, 036002 (2002); A. Dobado and J.R. Peláez, ibid. 65, 077502 (2002).

[21] G. Amorós, J. Bijnens, and P. Talavera, Nucl. Phys. B585, 293 (2000); B598, 665(E) (2001); B602, 87 (2001).

[22] J. Bijnens, G. Colangelo, and J. Gasser, Nucl. Phys. B427, 427 (1994). 